\author{
H. Bonel \\ K. A. Frei \\ L. Raio \\ M. Meyer-Wittkopf \\ L. Remonda \\ R. Wiest
}

\section{Prospective navigator-echo-based real-time triggering of fetal head movement for the reduction of artifacts}

Received: 22 May 2007

Revised: 30 August 2007

Accepted: 16 October 2007

Published online: 13 December 2007

(C) European Society of Radiology 2007

H. Bonel $(\bowtie)$

Institute of Diagnostic, Interventional and Pediatric Radiology,

University Hospital Berne-Inselspital, Freiburgstrasse,

3010 Bern, Switzerland

e-mail: bonel@gmx.ch

Tel : +41-31-6322435

Fax: +41-31-6324874

K. A. Frei · L. Raio ·

M. Meyer-Wittkopf

Women's' Hospital,

University of Berne,

Bern, Switzerland

L. Remonda $\cdot$ R. Wiest

Institute of Diagnostic and

Interventional Neuroradiology (DIN),

Inselspital, University of Berne,

Bern, Switzerland

\begin{abstract}
The purpose of this study was to evaluate the neuroimaging quality and accuracy of prospective real-time navigator-echo acquisition correction versus untriggered intrauterine magnetic resonance imaging (MRI) techniques. Twenty women in whom fetal motion artifacts compromised the neuroimaging quality of fetal MRI taken during the $28.7 \pm$ 4 week of pregnancy below diagnostic levels were additionally investigated using a navigator-triggered halfFourier acquired single-shot turbospin echo (HASTE) sequence.

Imaging quality was evaluated by two blinded readers applying a rating scale from 1 (not diagnostic) to 5 (excellent). Diagnostic criteria included depiction of the germinal matrix, grey and white matter, CSF, brain stem and cerebellum. Signal-difference-to-noise ratios (SDNRs) in the white matter and germinal zone were quantitatively evaluated. Imaging quality improved in $18 / 20$ patients using the navigator echo technique $(2.4 \pm 0.58$ vs. $3.65 \pm$ $0.73 \mathrm{SD}, \mathrm{p}<0.01$ for all evaluation
\end{abstract}

criteria). In $2 / 20$ patients fetal movement severely impaired image quality in conventional and navigated HASTE. Navigator-echo imaging revealed additional structural brain abnormalities and confirmed diagnosis in $8 / 20$ patients. The accuracy improved from $50 \%$ to $90 \%$. Average SDNR increased from $0.7 \pm 7.27$ to $19.83 \pm 15.71(p<0.01)$. Navigatorecho-based real-time triggering of fetal head movement is a reliable technique that can deliver diagnostic fetal MR image quality despite vigorous fetal movement.

Keywords Magnetic resonance imaging $\cdot$ Navigator $\cdot$ Artifacts · Motion · Fetal

\section{Introduction}

Over the past 2 decades, fetal magnetic resonance imaging (MRI) has evolved into an imaging modality that frequently provides better detail of the developing fetus than obstetric ultrasound $[1,2]$. Powerful gradients, advanced phasedarray coils and modern pulse sequences have significantly improved MRI image quality [1, 3, 4]. Like fetal ultrasound, fetal MRI is particularly good at identifying ventriculomegaly, lesions within the posterior fossa, callosal anomalies and abnormalities in cerebral myelination, migration and sulcation $[1,3,5-7]$. Compared to the real-time technique of obstetric ultrasound, however, MRI is constrained by motion artifacts caused by fetal movement $[1,7]$. Whereas the mother's 
Fig. 1 Images of a 37-year-old mother and her fetus at $215 / 7$ gestational weeks. True FISP images (a) show a lateral movement of the head on coronal planes; the dashed line indicates positioning of the navigator. (b) The effect of nontriggered (left column) versus triggered (right column) HASTE images in identical slice positions: motion artifacts are very pronounced using non-triggered images, while fetal brain anatomy is much clearer using these successfully navigated se-

quences. (c) Graph of navigator amplitude in the set of images provided by the MR scanner: the boxes at the top indicate the times of slice acquisition. In this patient, two phases of vigorous fetal movement lasting 2 and $1 / 2$ min prolonged the scan time to $7 \mathrm{~min}$ for the 18 slices obtained. The amplitude measures up to $3.7 \mathrm{~cm}$ in the medio-lateral direction
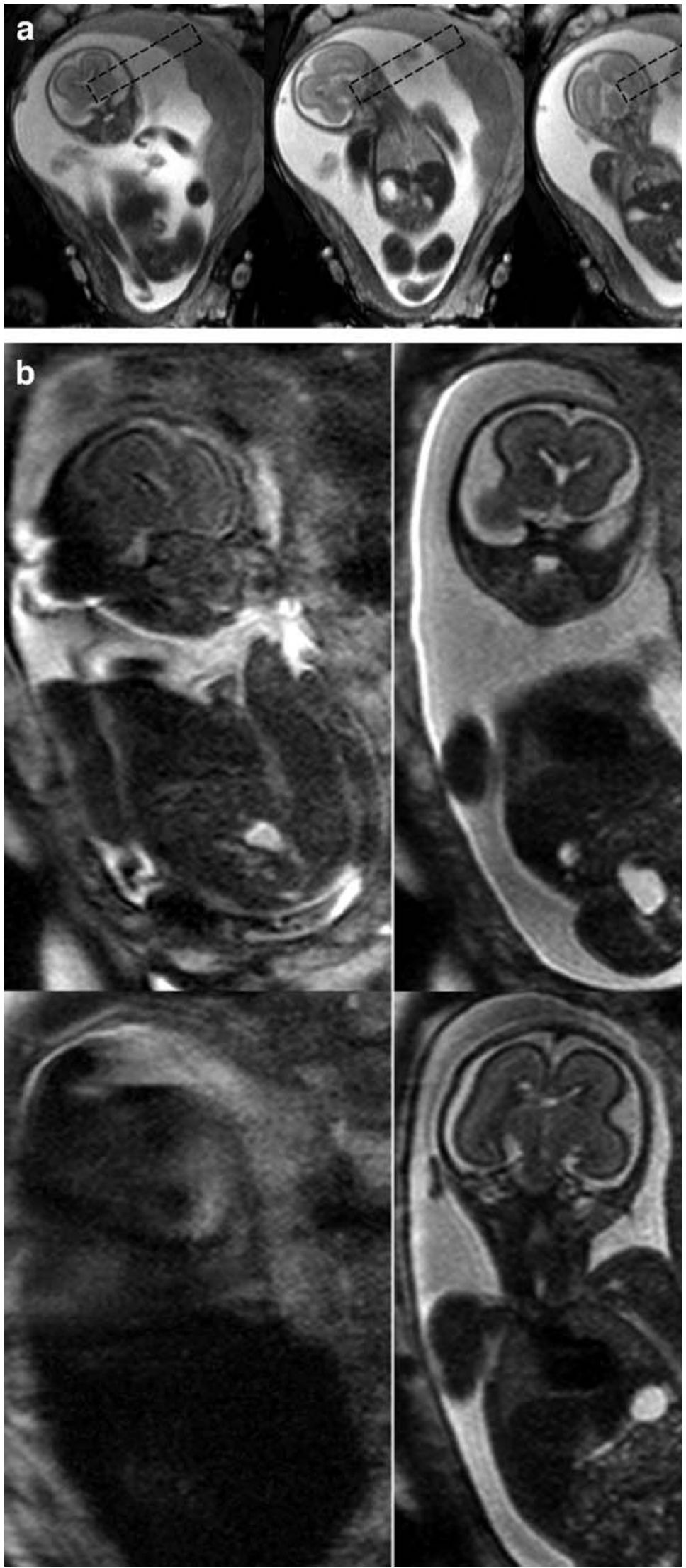
Fig. 1 (continued)

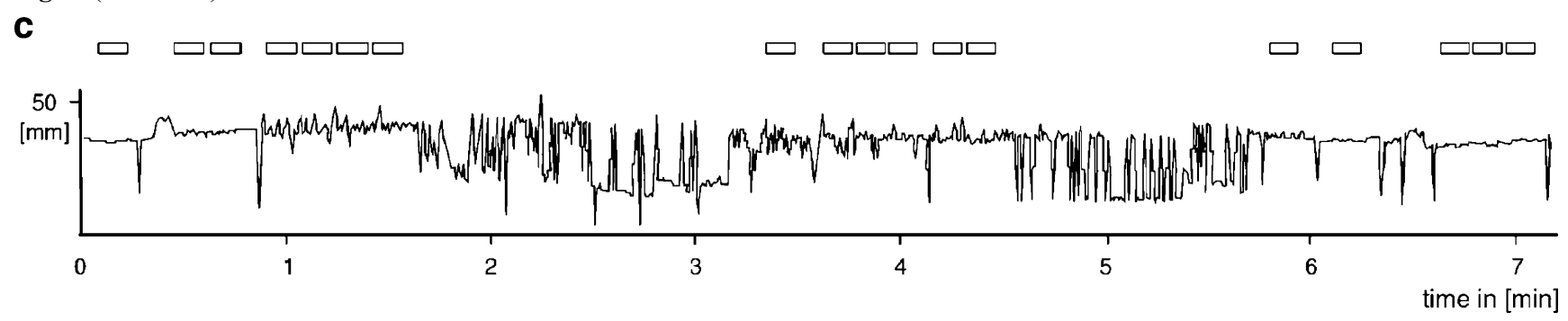

respiratory movements can be controlled by breathhold techniques, fetal movements will partially obscure the image quality of even numerous separately acquired slices of half Fourier single-shot turbo-spin echo sequences [6]. Moreover, it can be very tedious to examine such partially obscured stacks of slices that do not cover complete organs in one sequence.

One strategy to overcome such artifacts is prospective acquisition correction (PACE) done in real time during acquisition using a navigator commonly placed on the dome of the right diaphragm for abdominal MRI [8-10] or the head in adult patients $[11,12]$. In the present study, we applied this technique to the fetal head in a first step to control image acquisition by fetal movement in order to determine whether real time prospective acquisition correction improves the quality of fetal neuroimaging. The diagnostic image quality was assessed for non-navigated and navigated sequences in the same patient both quantitatively with signal-difference-to-noise ratios (SDNRs) [13] and also qualitatively on ordinal scales by two readers.

\section{Materials and methods}

Patients

Beginning in 2005, 88 consecutive mothers referred to our institution due to fetal abnormalities detected at obstetric ultrasound were prospectively examined by fetal MRI in order to (1) investigate the pathologies in greater detail and (2) to search for further structural changes not visible by ultrasound. In 20 of these 88 mothers (average maternal age $32.1 \pm 4.36$ years, range $24-42$ years; average gestational week $28.7 \pm 4$, range 21.7 to 37.7 weeks, including $2 / 20$ twin pregnancies), brain pathology was obscured by continuing vigorous fetal movement. For the evaluation of twin pregnancies only the fetus referred for further MRI evaluation was examined and then investigated for study purpose, because the other twin was usually not depicted by optimized planes. Additional navigated sequences were performed in these 88 patients to obtain MR images of diagnostic value.

This study was approved by the local ethics committee. All patients (i.e., the mothers) were informed in detail in advance of the examination and gave their written informed consent.

\section{MRI protocol}

MRI was performed with a commercially available 1.5-T MR scanner (Magnetom Sonata Maestro Class, Siemens Medical Systems, Erlangen, Germany) using a fourchannel body phased array coil combined with channels from the spine array coil adjacent to the fetus. Depending on patient comfort, patients were positioned supine or on the left lateral side. Intravenous contrast or sedative premedication was not used in any of the exams.

Our standard protocol uses T1-weighted fast-low angle shot $($ FLASH; TR $=85 \mathrm{~ms}, \mathrm{TE}=4.76 \mathrm{~ms}$, flip angle $=70$ degrees), T2-weighted half-Fourier acquired single-shot turbo-spin echo (HASTE; TR=1,260 ms, TE=84 ms), T1weighted inversion recovery $(\mathrm{TR}=9,470 \mathrm{~ms}, \mathrm{TE}=17 \mathrm{~ms})$ and T2-weighted true fast imaging with steady procession (FISP, TR=4.3 ms, TE $=1.86 \mathrm{~ms}$; all three gradients refocused) sequences with a slice thickness of $3 \mathrm{~mm}$ and one acquisition. The HASTE images were acquired in an interleaved order and take individually $1.02 \mathrm{~s}$ including sequence preparations. For true FISP sequences, parallel imaging was used in a subset of 16 patients with an acceleration factor of GRAPPA $=2$. For all sequences, the field of view (320-400 mm) and acquisition matrix (256$448 \mathrm{~mm}$ ) were adapted to the size of the mother to gain an in-plane resolution of $1.25 \times 1.25 \mathrm{~mm}$ or less. In addition, partial half Fourier was adjusted if a rectangular field of view was desired, but the spatial resolution was left unchanged. In all patients, axial and coronal images were acquired by HASTE sequences, which were evaluated for the purposes of this study. In addition, sagittal HASTE and/ or true FISP sequences were added depending on the indication. The number of slices was varied according to slice orientation and size of the fetus. Typically, fetal MRI examinations performed in our institution focus on two body regions. The scan time is generally $40 \mathrm{~min}$, but if there is any unforeseeable delay the examination is finally stopped after 50-min duration.

In case of severe motion artifacts in the T2-weighted HASTE sequence caused by vigorous fetal movement, a fast true FISP sequence was performed in the axial and/or 
Table 1 Levels of image quality obtained in 20 patients using navigated sequences (ranges shown in parentheses)

\begin{tabular}{lll}
\hline & Non-navigated & Navigated \\
\hline $\begin{array}{l}\text { Germinal zone/ } \\
\text { gray matter }\end{array}$ & $2 \pm 0.84(1.0$ to 4.0$)$ & $3.25 \pm 0.83(2.0$ to 4.0$)$ \\
White matter & $2.15 \pm 0.73(1.0$ to 4.0$)$ & $3.40 \pm 0.80(2.0$ to 4.0$)$ \\
CSF interspaces & $2.45 \pm 0.67(2.0$ to 4.0$)$ & $3.55 \pm 0.8(2.0$ to 5.0$)$ \\
Amniotic fluid & $2.45 \pm 0.67(1.0$ to 4.0$)$ & $3.75 \pm 0.62(2.0$ to 5.0$)$ \\
Brainstem & $2.45 \pm 0.74(1.0$ to 4.0$)$ & $3.60 \pm 0.73(2.0$ to 5.0$)$ \\
Cerebellum & $2.60 \pm 0.66(2.0$ to 4.0$)$ & $3.60 \pm 0.80(2.0$ to 5.0$)$ \\
Motion artifacts & $2.05 \pm 0.38(1.0$ to 3.0$)$ & $3.55 \pm 0.80(2.0$ to 5.0$)$ \\
Overall image & $2.40 \pm 0.58(2.0$ to 4.0$)$ & $3.65 \pm 0.73(2.0$ to 5.0$)$ \\
quality & & \\
\hline
\end{tabular}

Diagnostic levels of 3 or more were obtained in 18/20 patients. All evaluation criteria rated significantly better using navigated vs. nonnavigated sequences $(\mathrm{p}<0.01)$

coronal planes in order to characterize the preferred direction of fetal movement. The HASTE sequence was then repeated using navigator triggering and-for better comparison to the non-navigated sequence-the same parameters and number of slices. The $2 \mathrm{D}$ navigator sequence was positioned temporally or occipitally so as to cross the brain and skull, while comprising high contrast structures such as amniotic fluid and if possible the maternal abdominal wall or placenta. In addition, the sequence was oriented along the preferred direction of fetal movement as established in the preceding true FISP sequence. Positioning the navigator scan perpendicular to the squama of the temporal bone in a frontal orientation proved to be beneficial in most cases. Artifacts were controlled best if parts of the maternal anatomy, such as the uterus, placenta and/or abdominal wall, were included in the scan. If the navigated HASTE sequence was not able to acquire one anatomic image by the end of the first minute, the sequence was canceled and repeated after readjustment of the navigator. Without exception, the first readjustment allowed successful acquisition in all patients. Depending on fetal movement, acquisition time of the HASTE sequence was prolonged from a usual duration of $30 \mathrm{~s}$ for a typical average stack of 30 slices by an median of 3 min (range $1: 30$ to $7: 38$ min for a range of 26 to 40 slices, respectively) needed for the navigation of these images for the complete exam. The extra time for navigation needed per slice (average navigation time in one stack of images) therefore ranged between 4 and $12 \mathrm{~s}$ (median $6 \mathrm{~s}$ ) in our study.

\section{Image evaluation}

\section{Qualitative evaluation}

For qualitative evaluation, in a first session all images were evaluated by consensus of two board-certified neuroradiologists (RW and LR) who were blinded to image parameters, the sequences triggered, clinical findings and prior ultrasound reports. If consensus could not be reached by these readers, a third reader (HMB) supported and established consensus. The following eight criteria were evaluated on an ordinal scale regarding signal characteristics, potential dysmorphism and developmental anomalies ( $5=$ optimal diagnostic quality; $4=$ very good image quality; $3=$ diagnostic image quality, $2=$ image quality below diagnostic standards; $1=$ image quality too poor to correctly identify anatomy):

- Delineation of germinal zone and gray matter (according to the developmental stage) from white matter and the cerebro-spinal fluid (CSF)

- Delineation of white matter

- Delineation of internal and external CSF spaces

- Delineation of amniotic fluid adjacent to the skull

- Delineation of brain stem

- Delineation of cerebellum

- Severity of motion artifacts

- Overall image quality

A rating of 5 in the evaluation of motion artifacts implied the absence of notable artifacts; a rating of 5 for assessment of the brain stem implied optimal delineation of the brain stem with identification of the aqueduct and visible flow voids, which was best seen on sagittal planes. All read-

Table 2 Sensitivities, specificities and accuracies

\begin{tabular}{|c|c|c|c|c|}
\hline & MRI & Disease present & Disease absent & \\
\hline \multicolumn{5}{|c|}{ a. Sensitivities, specificities and accuracies of non-navigated HASTE sequences in the 88 patients } \\
\hline \multirow[t]{2}{*}{ Non-navigated } & Positive & 54 & 2 & Sensitivity: $84 \%$, specificity: $92 \%$, accuracy: $86 \%$ \\
\hline & Negative & 10 & 22 & \\
\hline \multicolumn{5}{|c|}{ b: Sensitivities, specificities and accuracies for the subgroup of 20 patients using both non-navigated and navigated sequences } \\
\hline \multirow[t]{2}{*}{ Non-navigated } & Positive & 0 & 0 & Sensitivity: $0 \%$, specificity: $100 \%$, accuracy: $50 \%$ \\
\hline & Negative & 10 & 10 & \\
\hline \multirow[t]{2}{*}{ Navigated } & Positive & 8 & 0 & Sensitivity $80 \%$, specificity: $100 \%$, accuracy: $90 \%$ \\
\hline & Negative & 2 & 10 & \\
\hline
\end{tabular}


Table 3 Diagnoses verified by navigated sequences

\begin{tabular}{ll}
\hline Diagnosis & Number of patients \\
\hline Partial agenesis of the corpus callosum & $\mathrm{n}=4$ \\
Polymicrogyria & $\mathrm{n}=2$ \\
Schizencephaly & $\mathrm{n}=2$ \\
\hline
\end{tabular}

ings were performed on a standard PACS workstation with two 3-megapixel monitors (Easyvision, Philips, Hamburg, Germany). Images were zoomed in to show the neurocranium at maximum size.

The amount of amniotic fluid was roughly assessed as (1) too little, (2) normal and (3) too much on an ordinal scale. Two months after the first evaluation and more than 6 months after the last MR examination, all examinations were evaluated again in a consensus session to determine in how many cases diagnosis would have been missed without navigated sequences. At this time, the evaluating neuroradiologists were aware of follow-up exams, current diagnosis and outcome. Postpartum MRI scans were available in 8 of the 20 patients, ultrasound exams of the head in 16 of the patients. In addition, all patients were followed clinically for at least 6 months to determine outcome and to correlate fetal to postpartum findings.

\section{Quantitative evaluation}

To evaluate the quality of imaging of the germinal zone versus the white matter, average signal intensities for the germinal zones and adjacent white matter were measured in four locations: left and right frontal brain, left and right post central gyri. To calculate signal-difference-to-noise ratios (SDNR) [13], the standard deviation of background noise of the respective slices was also assessed. Movement of the interpeduncular fossa was gauged and the amplitude of the navigator sequence quantified. The navigator amplitude was assessed in the set of images provided by the MR scanner (Fig. 1c) as a curve with times of acquisition.

All quantitative measurements were done by one reader (HMB) and cross-checked by a second reader (RW) on two 5-megapixel monitors using a standard PACS workstation (Easyvision, Philips, Hamburg, Germany) with images set to a zoom level of 1:1.

\section{Biostatistics}

The various qualitative criteria were tested for differences using the Wilcoxon matched-pairs signed-rank test. Quantitative data were tested for normal distribution and for differences with a t-test. A p-value of $<0.05$ was considered statistically significant.

\section{Results}

Qualitative evaluation

Navigated sequences significantly improved the diagnostic quality of the images in $18 / 20$ patients considering all criteria evaluated $(\mathrm{p}<0.01)$. In the two patients $(22 \mathrm{nd}$ and 28th gestational week, respectively) in whom the navigator did not significantly improve image quality, fetal head movement had a rotational component too great to be
Fig. 2 Images of a 24-year-old mother and her fetus at $375 / 7$ gestational weeks. Left column: two adjacent non-navigated paramedian sagittal slices; middle column: the same slices navigated; right column: postpartum images. A closed lip schizencephaly was diagnosed and an association with a polymicrogyria was postulated. Post-partum images confirm the diagnoses and more clearly show the polymicrogyria. This case was reported as a "missed diagnosis" (false negative, see Table 2) on non-navigated images

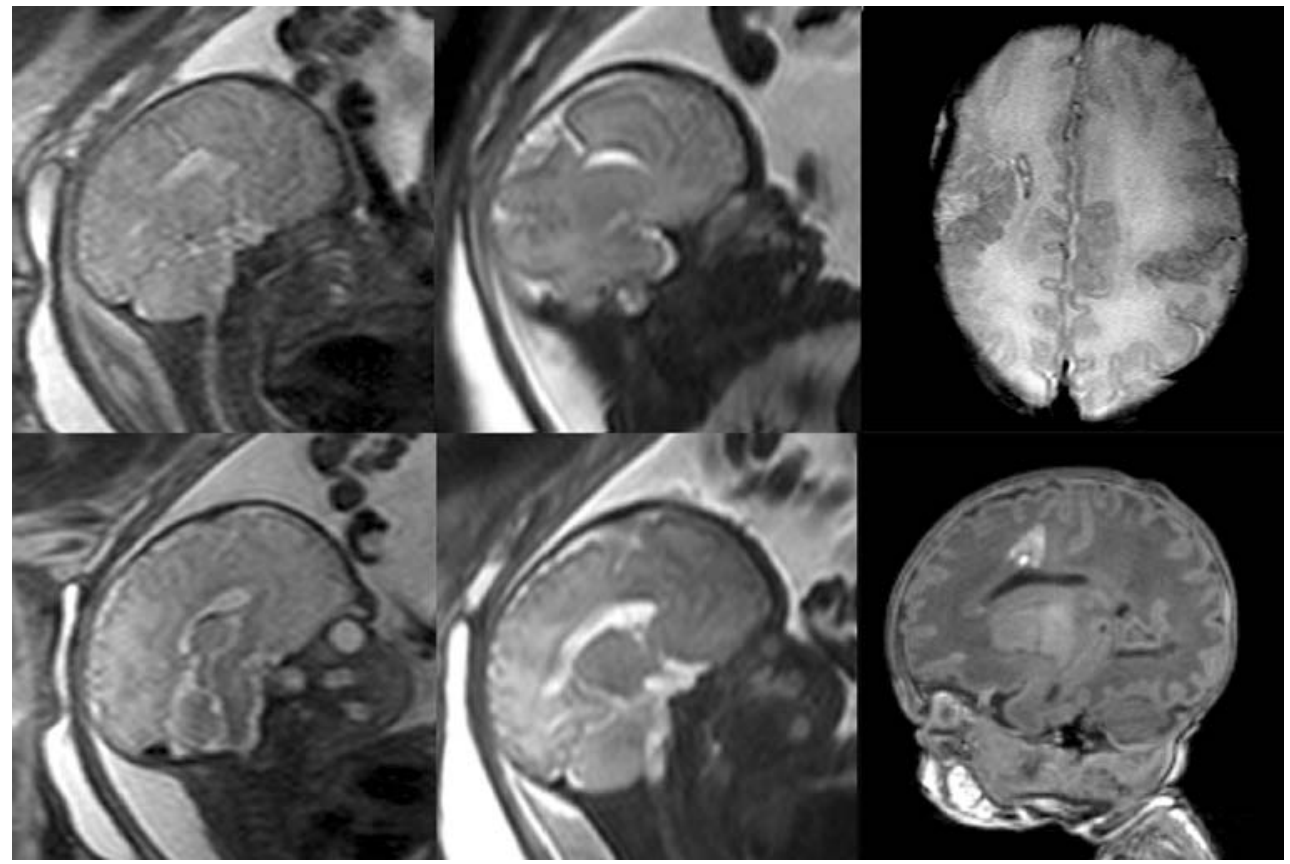



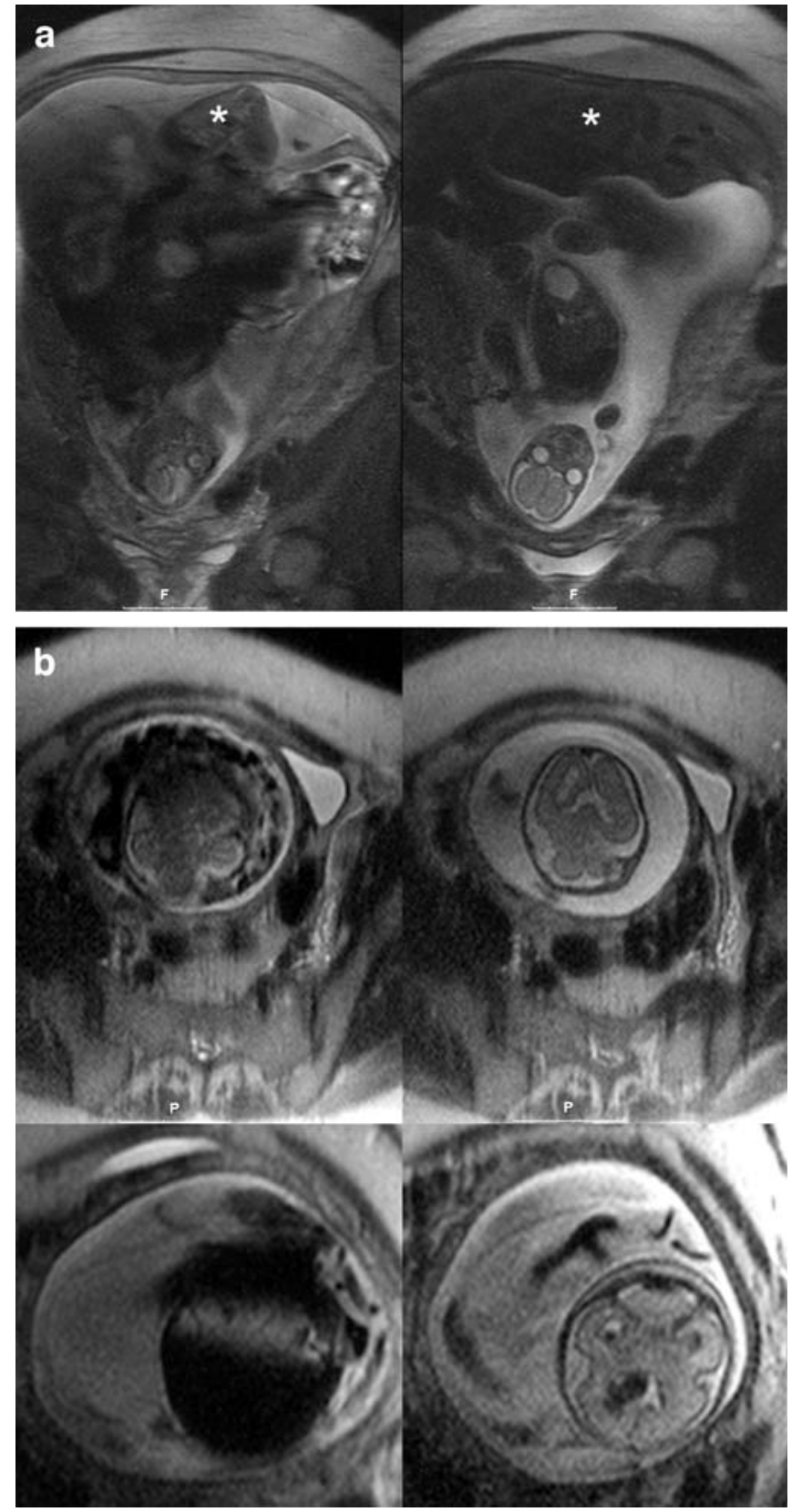

Fig. 3 Images of a 31-year-old mother at 22 gestational weeks with a twin pregnancy complicated by a feto-fetal transfusion syndrome. Coronal images show the donor in the fundus $(\mathbf{a}, *)$ and the head of the acceptor in the pelvis (b). Both twins moved constantly (a and $\mathbf{b}$, left images). When the navigator was applied to the head of the acceptor (on the right in $\mathbf{a}$ and $\mathbf{b}$ ), both coronal and axial images could be acquired with smaller motion artifacts, showing normal anatomy of the head (axial images, right upper image in b). Ten days later (axial images, lower row), the pregnancy was complicated by bleeding (navigated image on the lower right in $\mathbf{b}$ ), which is hardly visible on the non-navigated image on the left controlled by the $2 \mathrm{D}$ navigator used in this study. The MRI examination was not repeated in these patients. In $8 / 20$ patients, diagnosis would have been missed without triggering.

For the results of the qualitative assessment, see Table 1. All regions of the brain and CSF interspaces could be delineated and interpreted more accurately in navigated sequences (t-test, $\mathrm{p}<0.01$ ). The amount of amniotic fluid was rated as above average in three patients (gestational ages 27, 32 and 33 weeks); our rough assessment, however, found no correlation between the amount of amniotic fluid and fetal movement. There were 6 twin pregnancies in the original group of 88 patients. The movement artifacts were most pronounced in the two twin pregnancies with a early gestational age of 22 and 28 weeks requiring navigated HASTE sequences, and in comparison were less pronounced in the three pregnancies between 33 and 36 weeks and the one twin pregnancy in which the donor had already died because of a feto-fetal transfusion syndrome (these patients did not require navigated sequences). Table 2 gives sensitivities, specificities and accuracies; Table 3 presents newly discovered diagnoses in $8 / 20$ patients.

\section{Quantitative evaluation}

The navigator amplitude $(6.35 \pm 1.94 \mathrm{~cm}$, range 3.8 to $12 \mathrm{~cm}$ ) did not correlate with movement of the interpeduncular fossa $(3.89 \pm 0.86 \mathrm{~cm}$, range 2.4 to $6.2 \mathrm{~cm}, \mathrm{r}=0.42)$, which was significantly reduced on navigated sequences $(0.23 \pm 0.09$ vs. $3.89 \pm 0.86 \mathrm{~cm}, \mathrm{p}<0.01)$. Younger gestational age did not correlate with navigator amplitude $(\mathrm{r}=$ 0.12 ) or with overall image quality on non-navigated images $(\mathrm{r}=0.54)$, but it did correlate with movement of the interpeduncular fossa $(\mathrm{r}=0.98)$.

SDNR for distinguishing the germinal zone from the white matter was significantly better for navigated sequences ( $7.07 \pm 7.27$; range -6.33 to 34.71 ) than for non-navigated sequences $(19.83 \pm 15.71$, range 0.63 to 81.33 ; t-test, $\mathrm{p}<0.01)$.

Table 2 shows the sensitivities, specificities and accuracy of the navigated and non-navigated sequences. Table 3 presents specific diagnoses found only on navigated sequences, which were confirmed by follow-up.

\section{Discussion}

Since fetal MRI first evolved in the 1980s, movement artifacts caused by physiological motion of, e.g., the heart or diaphragm, as well by voluntary movement of the fetus, have interfered with imaging quality. In the beginning, sedation with benzodiazepines or paralysis, even with controversial substances such as curare, were used to overcome the limitation of this imaging technique [14]. In the $1990 \mathrm{~s}$, a major step forward was taken with the introduction of fast sequencing [6, 7]. Despite fast imaging 
sequences sedation is still a theme even in recent studies [15]. To date, however, triggering of fetal movement has not been used to improve intrauterine image quality.

Fetal movement can be so vigorous that even fast sequences by state-of-the art scanners, such as single-shot HASTE sequences, cannot depict fetal anatomy to diagnostic standards $(20 / 88=23 \%$ in our original patient cohort). We therefore applied navigator triggering, a well-known technique used especially to trigger movement of the diaphragm, to fetal MRI. This technique is of major interest for brain imaging because head movements are often more vigorous than those of other body regions, e.g., the pelvis, and the brain's size is ideal for triggering. Compared to other techniques, navigator imaging has the advantage of working without a monitoring device, such as a stretchable belt used for respiratory monitoring. Instead, a conventional cross-sliced navigator can be used to control the position of the fetal head.

Fetal movement was more common in the two early twin pregnancies of our cohort in which both twins were living. In addition, early gestational age correlates well with fetal head movement, which is best assessed by the movement of the interpeduncular fossa. Navigator amplitude, by contrast, seems to correlate less well with fetal movement, while other potential factors such as small fetal head or polyhydramnia did not correlate with fetal movement in our patient cohort. Of more relevance here could be factors such as fetal reaction to noise or MRI stretcher vibration, or severe cerebral abnormalities prohibiting fetal reactions to the uncommon situation in the scanner.

The application of a navigator echo sequence in fetal MRI requires optimized planning. Unlike the diaphragm, the fetal head can be moved in more than one direction, and its movements are irregular (Fig. 1c). We characterized fetal movement by doing a true FISP sequence right after the obscured HASTE sequence to localize the head and correctly apply the navigator sequence along the direction of preferred movement. For optimal imaging quality, true FISP and HASTE sequences require the triggering of matching slice positions (Fig. 1a and b). The irregular movement of the fetus does not necessarily pose a problem to the navigator sequence if the movement is in one direction and only varies in speed. Without proper localization of the navigator in relation to the fetal head, anatomic images cannot be acquired. Because the position is shown on the trigger monitor and indicated by an acoustic signal, the decision to stop sequences with unsuccessful triggering can be made ad hoc. In our series, navigated sequences that failed to acquire anatomical images within 1 min were stopped and the navigator readjusted. Without exception acquisition was successful on the first try after readjustment.
The sequence used for PACE has a low specific absorption rate, and no external device such as a cardiotocogram is needed on the body of the mother while in the scanner. We suggest that this technique therefore does not put the patient or his/her mother at a higher risk.

Although the number of patients in our series was limited, in 18 of the 20 patients all of the criteria evaluated revealed significantly improved neuroimaging quality and SDNRs. Fetal head movements are at times not translational, but have a rotational component, in flexion and/or extension movements along a transverse or sagittal axis. In $2 / 20$ patients, unusually complex movement patterns of the head with a strong rotational component hindered the acquisition of MR images of diagnostic quality. In conventional fetal MRI, the use of navigator triggering lowered the overall failure rate to only $2 / 88(2.3 \%)$. Applying an optimized strategy, the readers reported 8/20 (40\%) missed diagnoses comparing navigated and nonnavigated techniques (Figs. 2 and 3). In non-navigated sequences, the sensitivity of MRI in our patients was $84 \%$, the accuracy $86 \%$ (specificity 92\%). In the subgroup comparing non-navigated to navigated sequences, the sensitivity increased from $0 \%$ to $80 \%$, the accuracy from $50 \%$ to $90 \%$ (specificity unchanged, Table 2 ).

The present study has some limitations. First, we applied the technique in only a limited number of patients and focused on the fetal brain. Movement of fetal extremities, for example, is even more complex and would pose a great challenge for the $3 \mathrm{D}$ navigated technique. To date though we have not succeeded in acquiring diagnostic images of the limbs using this technique. Second, the correlation between navigator amplitude and movement of the interpeduncular fossa was poor. This can be attributed to the navigator's failure to monitor the full extent of fetal movement. The navigator echo, however, is acquired continuously, and momentary true FISP or single shot HASTE images could miss the most extreme positions.

\section{Conclusion}

In 18 of the 20 patients who underwent fetal MRI with navigator triggering, both imaging quality and SDNR improved. Moreover, additional diagnoses were found and later retrospectively confirmed in eight patients, and overall accuracy increased from $50 \%$ to $90 \%$. In our opinion, this result well justifies the longer acquisition time needed for navigated HASTE sequences. Further studies employing improved navigator sequences are needed to make this imaging modality available for all fetuses regardless of the intensity of fetal movement. 


\section{References}

1. Levine D, Barnes PD, Madsen JR, Li W, Edelman RR (1997) Fetal central nervous system anomalies: MR imaging augments sonographic diagnosis. Radiology 204:635-642

2. Prayer D, Brugger PC (2007) Investigation of normal organ development with fetal MRI. Eur Radiol 17(10):2458-2471

3. Coakley FV, Glenn OA, Qayyum A, Barkovich AJ, Goldstein R, Filly RA (2004) Fetal MRI: a developing technique for the developing patient. AJR 182(1):243-252

4. Baker PN, Johnson IR, Gowland PA, Freeman A, Adams V, Mansfield P (1994) Estimation of fetal lung volume using echo-planar magnetic resonance imaging. Obstet Gynecol 83(6):951-954

5. Glenn OA, Goldstein RB, Li KC, Young SJ, Norton ME, Busse RF, Goldberg JD, Barkovich AJ (2005) Fetal magnetic resonance imaging in the evaluation of fetuses referred for sonographically suspected abnormalities of the corpus callosum. J Ultrasound Med 24(6):791-804
6. Kubik-Huch RA, Wildermuth S, Cettuzzi L, Rake A, Seifert B, Chaoui R, Marincek B (2001) Fetus and uteroplacental unit: fast $\mathrm{mR}$ imaging with three-dimensional reconstruction and volumetry-feasibility study.

Radiology 219:567-573

7. Levine D, Hatabu H, Gaa J, Atkinson MW, Edelman RR (1996) Fetal anatomy revealed with fast MR sequences. AJR 167(4):905-908

8. Ehman RL, Felmlee JL (1989) Adaptive technique for high-definition MR imaging of moving structures.

Radiology 173(1):255-263

9. Kyriakos WE, Panych LP, Zientara GP, Jolesz FA (1997) Implementation of a reduced field-of-view method for dynamic MR imaging using navigator echoes. J Magn Reson Imaging 7(2):376-381

10. Klessen C, Asbach P, Kroencke TJ, Fischer T, Warmuth C, Stemmer A, Hamm B., Taupitz M (2005) Magnetic resonance imaging of the upper abdomen using a free-breathing T2weighted turbo spin echo sequence with navigator triggered prospective acquisition correction. J Magn Reson Imaging 21(5):576-582

11. Naganawa S, Koshikawa T, Fukatsu H, Ishigaki T, Maruyama K, Takizawa O (2004) Whole-brain vascular reactivity measured by fMRI using hyperventilation and breath-holding tasks: efficacy of 3D prospective acquisition correction (3D-PACE) for head motion. Eur Radiol 14(8):1484-1488
12. Barnwell JD, Smith JK, Castillo M (2007) Utility of navigator-prospective acquisition correction technique (PACE) for reducing motion in brain MR imaging studies. AJNR 28(4): 790-791

13. Wolff D, Balaban RS (1997) Assessing contrast on MR images. Radiology 202(1):25-29

14. Revel MP, Pons JC, Lelaidier C, Fournet P, Vial M, Musset D, Labrune M, Frydman R (1993) Magnetic resonance imaging of the fetus: a study of 20 cases performed without curarization. Prenat Diagn 13(9):775-799

15. Cannie M, Jani JC, De Keyzer F, Devlieger R, Van Schoubroeck D, Witters I, Marchal G, Dymarkowski S, Deprest JA (2006) Fetal body volume: use at MR imaging to quantify relative lung volume in fetuses suspected of having pulmonary hypoplasia. Radiology 241 : 847-853 